\title{
Accuracy of digital dental models using the low-cost DAVID laser scanner
}

\author{
Raphael Olszewski1,A-F, Joanna Szyper-Szczurowska ${ }^{2, B-F}$, Maciej Opach", ${ }^{3, B-F}$, \\ Piotr Bednarczyk ${ }^{4, B-F}$, Jan Zapala ${ }^{3, E, F}$, Stefan Szczepanik ${ }^{4, A-F}$ \\ 1 Department of Oral and Maxillofacial Surgery, Cliniques universitaires Saint-Luc, Catholic University of Louvain, Brussels, Belgium \\ 2 Department of Orthodontics, Jagiellonian University Medical College, Kraków, Poland \\ ${ }^{3}$ Department of Cranio-maxillofacial Surgery, Jagiellonian University Medical College, Kraków, Poland \\ ${ }^{4}$ Department of Metal Forming, AGH University of Science and Technology, Kraków, Poland \\ A - research concept and design; $\mathrm{B}$ - collection and/or assembly of data; $\mathrm{C}$ - data analysis and interpretation; \\ $D$ - writing the article; $E$ - critical revision of the article; $F$ - final approval of the article
}

\section{Address for correspondence}

Raphael Olszewski

E-mail: raphael.olszewski@uclouvain.be

\section{Funding sources}

None declared

Conflict of interest

None declared

Received on December 21, 2018

Review on January 22, 2019

Accepted on June 27, 2019

Published online on November 28, 2019

\section{Abstract}

Background. Accurate laser scanning of plaster casts using validated, low-cost hardware represents a key issue in $3 \mathrm{D}$ orthodontics.

Objectives. The aim of this study was to compare the accuracy of measurements taken from plaster casts (gold standard) with digital models of those casts created with a low-cost structural light DAVID laser scanner.

Material and methods. Five different measurements were taken on each of 14 plaster casts by 2 independent observers with an electronic caliper. The measurements were repeated 10 times on all 14 plaster casts by each observer, with a 1-week interval between each set of measurements. All 14 plaster casts were digitized using a low-cost DAVID SLS 3 laser scanner. The same 5 measurements were performed on each of the 3D virtual surface models of the 14 plaster casts by 2 independent observers using MeshLab software in a manner similar to that used with the digital caliper. The measurements were repeated 10 times by the 2 observers with 1 week between each set of measurements.

Results. The laser-scanned models were more accurate than the plaster cast models in defining measurements based on simple tooth fissures. The accuracy of measurements based on complex tooth fissures were equivalent for the 2 types of model. For measurements based on interproximal dental contacts, the 2 methods of measurement were similar and both were notably poor in terms of accuracy.

Conclusions. Three-dimensional virtual models obtained from the low-cost DAVID laser scanner can be used clinically, but only for certain types of measurements and indications.

Key words: orthodontics, laser scanner, digital dental models, plaster cast

Cite as

Olszewski R, Szyper-Szczurowska J, Opach M, Bednarczyk P,

Zapala J, Szczepanik S. Accuracy of digital dental models using the low-cost DAVID laser scanner. Adv Clin Exp Med. 2019;28(12):1647-1656. doi:10.17219/acem/110318

DOI

10.17219/acem/110318

\section{Copyright}

Copyright by Author(s)

This is an article distributed under the terms of the

Creative Commons Attribution 3.0 Unported (CC BY 3.0)

(https://creativecommons.org/licenses/by/3.0/) 


\section{Introduction}

Digital dental models are used in orthodontics because they are easy to store, save time and space and facilitate the sharing of information with colleagues over the internet. ${ }^{1}$ Digital models do not deteriorate over time. ${ }^{1}$ Laser scanners are accessible to clinicians through a digitization service, such as OrtoCad (Align Technology Inc., San Jose, USA) ${ }^{2,3}$ or "emodels" (GeoDigm Corp., Falcon Heights, USA), 2,3 through desktop laser scanners (i.e., 3Shape R500, 3Shape R700, 3Shape R1000, 3Shape R2000, Medianetx grande, Medianetx colori, DentaCore CS ULTRA, Dentaurum OrthoX, Maestro 3D, Imetric IScan D104i and GC Aadva Lab Scan ${ }^{4}$ ), through cone-beam computed tomography $(\mathrm{CT})^{5}$ and, recently, through intraoral laser scanners. ${ }^{6}$ All of these technologies are still very expensive and limit the spread of digital orthodontics to the wealthiest clinical practices and private hospitals. Moreover, desktop laser scanners present sufficient accuracy, so further improvement would not provide additional benefit for use in orthodontics. ${ }^{4}$ Nowak et al. ${ }^{4}$ concluded that research on laser scanners in orthodontics and orthognathic surgery should focus primarily on reducing time and cost. ${ }^{4}$

With the advent of the low-cost three-dimensional (3D) printing era, a number of companies have also attempted to develop low-cost laser scanners. Among the 3 types of low-cost laser scans currently available on the market, only the DAVID SLS 3 laser scanner provides a maximum resolution of $0.05 \%$ of the scanned object at a price of 3,275 USD (www.aniwaa.com/comparison/3d-scanners). Therefore, our objective for this study was to compare the accuracy of measurements taken from plaster casts (gold standard) with digital models obtained from the low-cost DAVID laser scanner. The null hypothesis is that the digital model is as accurate as a plaster-cast model, and that the low-cost DAVID laser scanner could be used clinically.

The DAVID SLS 3 laser scanner uses structural light and consists of a light projector, 2 detectors and a rotary table. A calibration kit for the device is also provided by the manufacturer. The projector projects 48 light structures onto the object to be scanned and the detectors analyze the deformation of these light structures on the scanned object, which is rotated on the rotary table.

\section{Material and methods}

Initially, 31 plaster-cast models from patients treated with orthodontics and orthognathic surgery and presenting maxillomandibular Angle class III discrepancies were used. From the 31 plaster casts, we discarded 17 with missing teeth in the areas of further distance measurements and selected the remaining 14 . The plaster casts were created in the same laboratory, and a similar length of time
Table 1. Definition of the measurements

\begin{tabular}{|c|c|}
\hline Name & Definition \\
\hline Measurement A & $\begin{array}{l}\text { Anterior width of the upper dental arch: } \\
\text { distance between the most lower points } \\
\text { of the transversal groove of the first upper } \\
\text { premolar teeth. }\end{array}$ \\
\hline Measurement B & $\begin{array}{l}\text { Posterior width of the upper dental arch: } \\
\text { distance between the points of intersection } \\
\text { of the transversal groove with the buccal groove } \\
\text { of the first upper permanent molar teeth. }\end{array}$ \\
\hline Measurement C & $\begin{array}{l}\text { Palatal width: distance between the intersection } \\
\text { points of the palatal groove with the gingival } \\
\text { margin of first upper permanent molar teeth } \\
\text { (Howe et al. }{ }^{2} \text { ). }\end{array}$ \\
\hline Measurement D & $\begin{array}{l}\text { Anterior width of the lower dental arch: distance } \\
\text { between the vestibular contact points of the first } \\
\text { and the second lower premolars. }\end{array}$ \\
\hline Measurement E & $\begin{array}{l}\text { Posterior width of the lower dental arch: distance } \\
\text { between the distal and lingual cusp tips of right } \\
\text { and left mandibular permanent first molars. }\end{array}$ \\
\hline
\end{tabular}

separated the alginate impression from casting. ${ }^{2}$ Two calibrated observers participated in this study. Observer \#1 was an experienced orthodontist, while observer \#2 was a maxillofacial surgeon. The 2 examiners were calibrated by collaborating on 2 sample cases of plaster casts and 2 sample cases of laser-scanned casts. ${ }^{2}$ The measurements were directly compared and discussed until final definition. $^{2}$

Five measurements (A-E) (Table 1) were performed on each of the 14 plaster casts by the 2 independent observers using an electronic caliper (OTLT, Otelo, Saint-Ouenl'Aumône, France) with a measurement error of $0.02 \mathrm{~mm}$. The measurements were repeated on all 14 of the plaster casts 10 times each by the 2 observers, with a 1 -week interval between each set of measurements.

All plaster casts (\#1 to \#14) were also digitized using a DAVID SLS 3 laser scanner v. 4.5.3 (DAVID; Antonius Köster, Meschede, Germany). The scanning angle was $36^{\circ}$. Each digitalized model was created from 10 measurements (a full rotation of the table is $360^{\circ}$ ). The cloud of points was then analyzed with DAVID SLS 3 software v. 4.5.3 (Antonius Köster). A 3D virtual surface model (.obj file) of each plaster cast was saved for further measurements by the 2 observers. Five measurements (A-E) (Table 1) were performed on each of the $3 \mathrm{D}$ virtual surface models of the 14 plaster casts by 2 independent observers with MeshLab software (v. 1.3.2) (Consiglio Nazionale delle Ricerche - CNR, Rome, Italy) in a manner analogous to that employed with the digital caliper. The measurements were repeated on all of the $3 \mathrm{D}$ virtual surface models of the 14 plaster casts 10 times by 2 independent observers. A 1-week period of time elapsed between each set of measurements. Measurement A on model \#4 was impossible to perform because one of the premolars was missing on the plaster-cast model. Palatal width definition was proposed according to the study by Howe et al. ${ }^{7}$ 
Figure 1 shows the measurements performed on the plaster casts, while Fig. 2 and 3 illustrate the measurements performed on the laser-scanned virtual 3D models of the plaster casts.

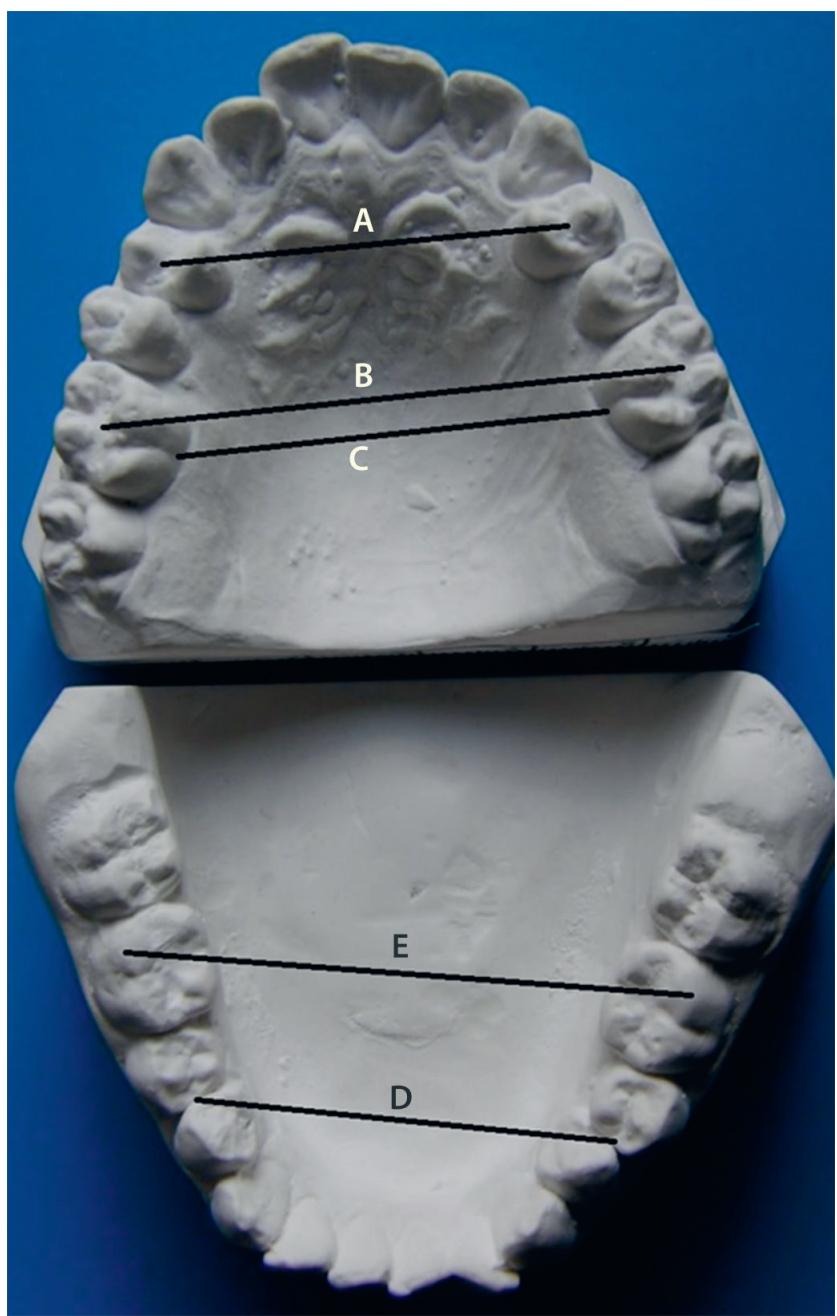

Fig. 1. Measurements A-C performed on the plaster cast of the upper maxilla. Measurements $D$ and $E$ performed on the plaster cast of the mandible

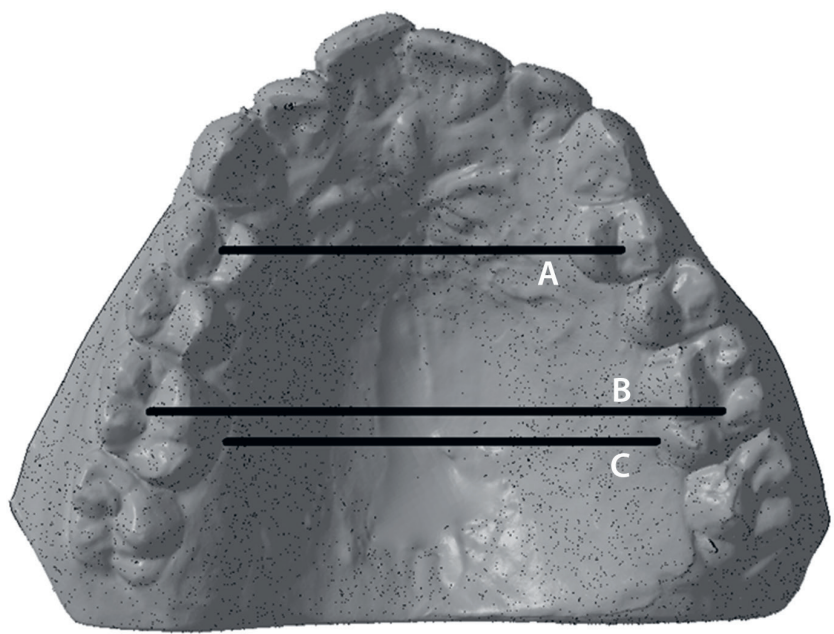

Fig. 2. Measurements A-C performed on the virtual 3D model of the laser-scanned upper maxilla plaster cast

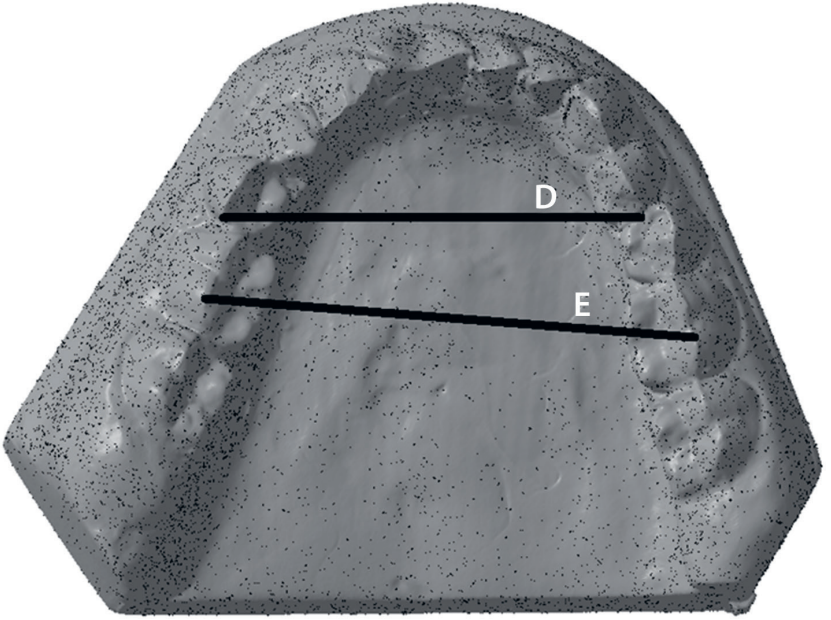

Fig. 3. Measurements $D$ and E performed on the virtual 3D model of the laser-scanned mandibular plaster cast

\section{Results}

For statistical analysis, we assumed that the population presented a normal distribution. The populations represent 2 small groups of 14 elements, each consisting of measurements (Table 1) performed by observer \#1 and observer \#2. Table 2 (observer \#1) and Table 3 (observer \#2) show the pairs of measurements obtained from the 14 plaster casts with the minimum and maximum values, the difference between these values, the mean values, and their standard deviation (SD). Table 4 shows a comparison of the measurements taken by observers \#1 and \#2 and the confidence interval $(95 \% \mathrm{CI})$ with $\alpha=0.05$. Table 5 shows the Cis for the differences between the measurements observed by observer \#1 and observer \#2, according to the type of method (caliper vs $3 \mathrm{D}$ virtual model) and the type of measurement (A-E).

The CIs for the measurements performed by observer \#1 (orthodontist) on the plaster casts and on the digital models are $0.069-0.196 \mathrm{~mm}$ and $0.057-0.329 \mathrm{~mm}$, respectively. The CIs for the measurements performed by observer \#2 (maxillofacial surgeon) on the plaster casts and on the digital models are $0.054-0.408 \mathrm{~mm}$ and $0.136-0.429 \mathrm{~mm}$, respectively.

Measurement A was based on the anatomical definition of a simple tooth fissure. The laser-scanned models were more accurate than the plaster-cast models in defining measurement A (Table 5). Measurement B was based on the anatomical definition of a complex tooth fissure. Measurement $C$ was based on the intersection between 2 different structures, such as a tooth fissure and the impression of the palatal gingiva on the tooth. The accuracy of measurements $B$ and $C$ was equivalent for the laserscanned and the plaster models. Measurement $\mathrm{D}$ was based on interproximal dental contacts. Measurement E was based on the tips of cusps (curvature areas). Measurements $\mathrm{D}$ and $\mathrm{E}$ were equivalent and provided notably poor accuracy. Our findings related to measurements D and E were in accordance with the literature. ${ }^{2}$ 
Table 2. Measurements performed by observer \#1

\begin{tabular}{|c|c|c|c|c|c|c|c|c|c|c|}
\hline \multirow[b]{2}{*}{$\begin{array}{l}\text { Measurement } \\
\text { name }\end{array}$} & \multicolumn{5}{|c|}{$\begin{array}{l}\text { Caliper measurement } \\
\text { plaster cast }\end{array}$} & \multicolumn{5}{|c|}{$\begin{array}{l}\text { 3D virtual model measurement } \\
\text { laser scanner }\end{array}$} \\
\hline & $\begin{array}{c}\text { maximal } \\
\text { value } \\
(\mathrm{max}) \\
{[\mathrm{mm}]}\end{array}$ & $\begin{array}{l}\text { minimal } \\
\text { value } \\
\text { (min) } \\
\text { [mm] }\end{array}$ & $\begin{array}{c}\text { difference } \\
\text { [max-min] } \\
{[\mathrm{mm}]}\end{array}$ & $\begin{array}{l}\text { mean } \\
\text { value } \\
{[\mathrm{mm}]}\end{array}$ & $\begin{array}{c}\text { standard } \\
\text { deviation } \\
{[\mathrm{mm}]}\end{array}$ & $\begin{array}{c}\text { maximal } \\
\text { value } \\
\text { (max) } \\
\text { [mm] }\end{array}$ & $\begin{array}{l}\text { minimal } \\
\text { value } \\
\text { (min) } \\
{[\mathrm{mm}]}\end{array}$ & $\begin{array}{c}\text { difference } \\
\text { [max-min] } \\
{[\mathrm{mm}]}\end{array}$ & $\begin{array}{l}\text { mean } \\
\text { value } \\
{[\mathrm{mm}]}\end{array}$ & $\begin{array}{c}\text { standard } \\
\text { deviation } \\
{[\mathrm{mm}]}\end{array}$ \\
\hline $\mathrm{m} 01 \mathrm{~A}$ & 33.02 & 32.44 & 0.58 & 32.81 & 0.17 & 33.35 & 32.58 & 0.77 & 32.92 & 0.30 \\
\hline $\mathrm{m} 01 \mathrm{C}$ & 34.76 & 33.50 & 0.26 & 34.43 & 0.37 & 35.60 & 34.77 & 0.83 & 35.27 & 0.23 \\
\hline m01 D & 33.63 & 33.01 & 0.62 & 33.29 & 0.18 & 33.80 & 33.24 & 0.56 & 33.49 & 0.20 \\
\hline $\mathrm{m} 01 \mathrm{E}$ & 43.62 & 42.39 & 1.23 & 42.93 & 0.37 & 43.63 & 42.76 & 0.87 & 43.20 & 0.26 \\
\hline $\mathrm{m02} \mathrm{A}$ & 38.86 & 38.22 & 0.64 & 38.51 & 0.21 & 39.07 & 38.33 & 0.74 & 38.70 & 0.27 \\
\hline $\mathrm{m} 02 \mathrm{~B}$ & 51.97 & 51.19 & 0.78 & 51.78 & 0.22 & 53.11 & 52.11 & 1.00 & 52.46 & 0.28 \\
\hline $\mathrm{m} 02 \mathrm{D}$ & 37.59 & 36.39 & 1.20 & 37.00 & 0.34 & 38.77 & 37.97 & 0.80 & 38.44 & 0.26 \\
\hline $\mathrm{m02} \mathrm{E}$ & 50.65 & 49.49 & 1.16 & 50.11 & 0.32 & 50.33 & 49.52 & 0.81 & 49.90 & 0.26 \\
\hline $\mathrm{m03} \mathrm{A}$ & 36.28 & 35.62 & 0.66 & 36.05 & 0.22 & 37.05 & 35.73 & 1.32 & 36.52 & 0.36 \\
\hline m03 B & 44.92 & 44.24 & 0.68 & 44.50 & 0.23 & 45.38 & 44.46 & 0.92 & 44.89 & 0.26 \\
\hline $\mathrm{m03} C$ & 32.53 & 31.02 & 1.51 & 32.09 & 0.42 & 33.94 & 33.22 & 0.72 & 33.50 & 0.24 \\
\hline m03 D & 35.80 & 34.31 & 1.49 & 34.77 & 0.40 & 36.89 & 35.12 & 1.77 & 35.94 & 0.60 \\
\hline $\mathrm{m03} \mathrm{E}$ & 45.88 & 44.91 & 0.97 & 45.36 & 0.31 & 46.52 & 45.05 & 1.47 & 45.53 & 0.43 \\
\hline $\mathrm{m04} \mathrm{A}$ & - & - & - & - & - & - & - & - & - & - \\
\hline m04 B & 46.88 & 45.51 & 1.37 & 46.26 & 0.40 & 47.00 & 45.99 & 1.01 & 46.43 & 0.29 \\
\hline $\mathrm{m04C}$ & 34.40 & 32.53 & 1.87 & 33.54 & 0.57 & 34.89 & 34.00 & 0.89 & 34.44 & 0.36 \\
\hline m04 D & 35.66 & 34.11 & 1.55 & 34.88 & 0.44 & 36.89 & 36.14 & 0.75 & 36.41 & 0.19 \\
\hline m05 A & 29.90 & 29.04 & 0.86 & 29.48 & 0.29 & 29.75 & 29.01 & 0.74 & 29.54 & 0.22 \\
\hline m05 B & 42.89 & 41.70 & 1.19 & 42.27 & 0.38 & 43.27 & 42.23 & 1.04 & 42.76 & 0.34 \\
\hline $\mathrm{m05C}$ & 32.90 & 31.74 & 1.16 & 32.29 & 0.36 & 33.87 & 32.83 & 1.04 & 33.36 & 0.32 \\
\hline m05 D & 31.44 & 30.12 & 1.32 & 30.58 & 0.37 & 31.90 & 30.59 & 1.31 & 31.14 & 0.45 \\
\hline $\mathrm{m05} \mathrm{E}$ & 41.84 & 40.64 & 1.20 & 41.24 & 0.41 & 42.26 & 41.36 & 0.90 & 41.70 & 0.32 \\
\hline $\mathrm{m06} \mathrm{A}$ & 35.83 & 34.81 & 1.02 & 35.26 & 0.30 & 35.93 & 35.03 & 0.90 & 35.45 & 0.30 \\
\hline m06 B & 51.75 & 51.11 & 0.64 & 51.41 & 0.20 & 52.07 & 51.32 & 0.75 & 51.68 & 0.27 \\
\hline $\mathrm{m06C}$ & 39.08 & 38.15 & 0.93 & 38.58 & 0.28 & 40.08 & 38.93 & 1.15 & 39.43 & 0.34 \\
\hline m06 D & 37.58 & 36.36 & 1.22 & 37.07 & 0.33 & 38.92 & 37.72 & 1.20 & 38.22 & 0.40 \\
\hline $\mathrm{m} 06 \mathrm{E}$ & 51.59 & 50.42 & 1.17 & 51.15 & 0.28 & 52.20 & 51.23 & 0.97 & 51.67 & 0.29 \\
\hline $\mathrm{m} 07 \mathrm{~A}$ & 30.04 & 29.68 & 0.36 & 29.93 & 0.10 & 31.47 & 30.08 & 1.39 & 30.56 & 0.45 \\
\hline m07 B & 39.98 & 39.42 & 0.56 & 39.69 & 0.20 & 40.46 & 38.99 & 1.47 & 39.74 & 0.49 \\
\hline $\mathrm{m07C}$ & 30.32 & 28.84 & 1.48 & 29.49 & 0.44 & 31.68 & 30.54 & 1.14 & 31.06 & 0.33 \\
\hline m07 D & 35.11 & 34.14 & 0.97 & 34.56 & 0.33 & 35.86 & 34.32 & 1.54 & 35.00 & 0.46 \\
\hline $\mathrm{m07} \mathrm{E}$ & 46.66 & 45.98 & 0.68 & 46.33 & 0.26 & 48.29 & 47.07 & 1.22 & 47.58 & 0.43 \\
\hline $\mathrm{m08} \mathrm{A}$ & 34.89 & 33.67 & 1.22 & 34.23 & 0.39 & 34.93 & 34.28 & 0.65 & 34.55 & 0.23 \\
\hline m08 B & 44.75 & 43.86 & 0.89 & 44.39 & 0.29 & 45.01 & 44.09 & 0.92 & 44.52 & 0.22 \\
\hline $\mathrm{m08C}$ & 35.18 & 33.22 & 1.96 & 34.20 & 0.59 & 36.63 & 35.87 & 0.76 & 36.30 & 0.28 \\
\hline m08 D & 32.45 & 31.26 & 1.19 & 32.13 & 0.32 & 33.83 & 32.08 & 1.75 & 32.84 & 0.55 \\
\hline $\mathrm{m08E}$ & 45.40 & 43.47 & 1.93 & 44.41 & 0.45 & 45.46 & 44.64 & 0.82 & 45.10 & 0.26 \\
\hline m09 A & 37.05 & 36.09 & 0.96 & 36.42 & 0.26 & 37.20 & 35.63 & 1.57 & 36.47 & 0.38 \\
\hline m09 B & 50.70 & 49.61 & 1.09 & 50.23 & 0.31 & 50.36 & 49.10 & 1.26 & 49.93 & 0.35 \\
\hline
\end{tabular}


Table 2. Measurements performed by observer \#1 - cont.

\begin{tabular}{|c|c|c|c|c|c|c|c|c|c|c|}
\hline \multirow[b]{2}{*}{$\begin{array}{l}\text { Measurement } \\
\text { name }\end{array}$} & \multicolumn{5}{|c|}{$\begin{array}{l}\text { Caliper measurement } \\
\text { plaster cast }\end{array}$} & \multicolumn{5}{|c|}{$\begin{array}{l}\text { 3D virtual model measurement } \\
\text { laser scanner }\end{array}$} \\
\hline & $\begin{array}{c}\text { maximal } \\
\text { value } \\
\text { (max) } \\
\text { [mm] }\end{array}$ & $\begin{array}{l}\text { minimal } \\
\text { value } \\
\text { (min) } \\
\text { [mm] }\end{array}$ & $\begin{array}{c}\text { difference } \\
\text { [max-min] } \\
{[\mathrm{mm}]}\end{array}$ & $\begin{array}{l}\text { mean } \\
\text { value } \\
{[\mathrm{mm}]}\end{array}$ & $\begin{array}{c}\text { standard } \\
\text { deviation } \\
{[\mathrm{mm}]}\end{array}$ & $\begin{array}{l}\text { maximal } \\
\text { value } \\
\text { (max) } \\
{[\mathrm{mm}]}\end{array}$ & $\begin{array}{c}\text { minimal } \\
\text { value } \\
\text { (min) } \\
{[\mathrm{mm}]}\end{array}$ & $\begin{array}{c}\text { difference } \\
\text { [max-min] } \\
{[\mathrm{mm}]}\end{array}$ & $\begin{array}{l}\text { mean } \\
\text { value } \\
{[\mathrm{mm}]}\end{array}$ & $\begin{array}{c}\text { standard } \\
\text { deviation } \\
{[\mathrm{mm}]}\end{array}$ \\
\hline m09C & 38.23 & 37.46 & 0.77 & 37.87 & 0.26 & 39.49 & 38.57 & 0.92 & 39.11 & 0.32 \\
\hline m09 D & 31.66 & 30.50 & 1.16 & 31.03 & 0.37 & 32.57 & 31.82 & 0.75 & 32.16 & 0.22 \\
\hline m09 E & 42.08 & 40.69 & 1.39 & 41.38 & 0.41 & 44.97 & 44.10 & 0.87 & 44.51 & 0.26 \\
\hline $\mathrm{m} 10 \mathrm{~A}$ & 39.92 & 39.35 & 0.57 & 39.59 & 0.18 & 41.21 & 40.34 & 0.87 & 40.82 & 0.25 \\
\hline m10 B & 49.83 & 49.17 & 0.66 & 49.44 & 0.20 & 51.08 & 49.96 & 1.12 & 50.38 & 0.29 \\
\hline $\mathrm{m} 10 \mathrm{C}$ & 38.73 & 37.11 & 1.62 & 37.84 & 0.47 & 38.66 & 37.79 & 0.87 & 38.32 & 0.28 \\
\hline m10 D & 35.21 & 33.08 & 2.13 & 34.11 & 0.62 & 36.42 & 34.91 & 1.51 & 35.43 & 0.44 \\
\hline $\mathrm{m} 10 \mathrm{E}$ & 45.88 & 45.18 & 0.70 & 45.55 & 0.21 & 46.24 & 45.26 & 0.98 & 45.82 & 0.33 \\
\hline $\mathrm{m} 11 \mathrm{~A}$ & 34.85 & 33.37 & 1.48 & 33.95 & 0.40 & 34.94 & 33.98 & 0.96 & 34.39 & 0.35 \\
\hline $\mathrm{m} 11 \mathrm{~B}$ & 45.49 & 44.49 & 1.00 & 45.08 & 0.34 & 46.61 & 45.37 & 1.24 & 45.71 & 0.36 \\
\hline $\mathrm{m} 11 \mathrm{C}$ & 32.48 & 31.51 & 0.97 & 31.92 & 0.29 & 33.44 & 31.81 & 1.63 & 32.27 & 0.47 \\
\hline m11 D & 31.42 & 30.17 & 1.25 & 30.81 & 0.42 & 32.82 & 31.38 & 1.44 & 32.01 & 0.45 \\
\hline $\mathrm{m} 11 \mathrm{E}$ & 44.42 & 43.13 & 1.29 & 43.78 & 0.46 & 44.02 & 43.02 & 1.00 & 43.56 & 0.37 \\
\hline $\mathrm{m} 12 \mathrm{~A}$ & 45.73 & 45.18 & 0.55 & 45.47 & 0.14 & 46.44 & 45.72 & 0.72 & 46.06 & 0.23 \\
\hline m12 B & 55.73 & 54.95 & 0.78 & 55.29 & 0.24 & 56.32 & 55.49 & 0.83 & 55.84 & 0.26 \\
\hline $\mathrm{m} 12 \mathrm{C}$ & 43.27 & 41.71 & 1.56 & 42.65 & 0.50 & 44.23 & 43.05 & 1.18 & 43.42 & 0.42 \\
\hline m12 D & 42.83 & 41.35 & 1.48 & 42.16 & 0.42 & 43.17 & 41.98 & 1.19 & 42.58 & 0.31 \\
\hline $\mathrm{m} 12 \mathrm{E}$ & 57.37 & 56.19 & 1.18 & 56.69 & 0.32 & 57.51 & 56.22 & 1.29 & 56.78 & 0.40 \\
\hline m13 A & 32.34 & 31.43 & 0.91 & 31.73 & 0.27 & 32.52 & 30.81 & 1.71 & 31.78 & 0.51 \\
\hline $\mathrm{m} 13 \mathrm{~B}$ & 45.50 & 44.57 & 0.93 & 45.08 & 0.29 & 45.24 & 44.57 & 0.67 & 44.81 & 0.25 \\
\hline $\mathrm{m} 13 \mathrm{C}$ & 33.04 & 31.91 & 1.13 & 32.29 & 0.31 & 34.33 & 32.95 & 1.38 & 33.73 & 0.34 \\
\hline m13 D & 36.63 & 35.39 & 1.24 & 35.77 & 0.33 & 37.24 & 35.71 & 1.53 & 36.63 & 0.44 \\
\hline $\mathrm{m} 13 \mathrm{E}$ & 53.15 & 51.96 & 1.19 & 52.48 & 0.33 & 53.62 & 52.06 & 1.56 & 52.92 & 0.53 \\
\hline $\mathrm{m} 14 \mathrm{~A}$ & 33.93 & 32.30 & 1.63 & 32.99 & 0.39 & 34.43 & 33.65 & 0.78 & 34.08 & 0.24 \\
\hline m14 B & 45.39 & 44.30 & 1.09 & 44.81 & 0.31 & 45.57 & 44.74 & 0.83 & 45.15 & 0.25 \\
\hline $\mathrm{m} 14 \mathrm{C}$ & 32.66 & 31.03 & 1.63 & 31.83 & 0.52 & 34.10 & 32.66 & 1.44 & 33.29 & 0.47 \\
\hline m14 D & 35.89 & 34.70 & 1.19 & 35.41 & 0.36 & 37.51 & 36.22 & 1.29 & 36.67 & 0.42 \\
\hline m14 E & 48.78 & 46.99 & 1.79 & 48.05 & 0.52 & 48.78 & 46.11 & 2.67 & 47.16 & 0.87 \\
\hline
\end{tabular}

\section{Discussion}

The measurements taken by the orthodontist were more accurate than those taken by the maxillofacial surgeon, possibly because of personal experience and the clinical use of plaster casts in daily orthodontic practice. However, the mean values differ between the observers and the methods. The difference in measurements observed between both observers and both methods may be related to the observer's aptitude of correctly selecting landmarks which correspond to their theoretical definition. This selection may be influenced by 1) the subjective interpretation of the landmark's definition, 2) the quality of the occlusal surfaces and the interproximal contact points of the patient's teeth,
3) the quality of the impression obtained, 4) the type of material used for the plaster cast, 5) the color of the plaster cast, 6) the color of the 3D virtual rendering on the computer screen, and 7) 3D manipulation of the digital cast in the software (zooming, rotating and selecting views). ${ }^{8}$ Measurements A-C performed on the digital models (Table 5) were included below the threshold difference of $1.5 \mathrm{~mm}$ which was suggested by Profitt as a limiting value for clinical significance. ${ }^{9}$ The majority of measurements $\mathrm{D}$ and $\mathrm{E}$ were above the threshold difference of $1.5 \mathrm{~mm}$ for both methods (Table 5). Therefore, measurements D and E should be discarded from further comparative studies regarding the accuracy of laser-scanned and plaster-cast models. 
Table 3. Measurements performed by observer \#2

\begin{tabular}{|c|c|c|c|c|c|c|c|c|c|c|}
\hline \multirow{2}{*}{$\begin{array}{l}\text { Measurement } \\
\text { name }\end{array}$} & \multicolumn{10}{|c|}{ Observer \#2 } \\
\hline & \multicolumn{5}{|c|}{$\begin{array}{l}\text { caliper measurement } \\
\text { plaster cast }\end{array}$} & \multicolumn{5}{|c|}{$\begin{array}{l}\text { 3D virtual model measurement } \\
\text { laser scanner }\end{array}$} \\
\hline $\mathrm{m} 01 \mathrm{~A}$ & 33.84 & 33.18 & 0.66 & 33.39 & 0.22 & 33.12 & 32.51 & 0.61 & 32.85 & 0.16 \\
\hline $\mathrm{m} 01 \mathrm{~B}$ & 47.48 & 46.94 & 0.54 & 47.22 & 0.19 & 47.69 & 47.24 & 0.45 & 47.46 & 0.18 \\
\hline $\mathrm{m} 01 \mathrm{C}$ & 34.50 & 33.57 & 0.93 & 34.03 & 0.31 & 35.82 & 35.07 & 0.75 & 35.45 & 0.21 \\
\hline m01 D & 35.33 & 34.22 & 1.11 & 34.78 & 0.39 & 35.35 & 34.72 & 0.63 & 35.12 & 0.18 \\
\hline $\mathrm{m} 01 \mathrm{E}$ & 45.93 & 45.35 & 0.58 & 45.66 & 0.21 & 46.17 & 45.48 & 0.69 & 45.84 & 0.20 \\
\hline $\mathrm{m02} \mathrm{A}$ & 40.94 & 39.18 & 1.76 & 40.04 & 0.45 & 39.45 & 38.56 & 0.89 & 38.86 & 0.28 \\
\hline $\mathrm{m} 02 \mathrm{~B}$ & 52.23 & 51.85 & 0.38 & 52.11 & 0.11 & 52.56 & 51.65 & 0.91 & 52.27 & 0.24 \\
\hline $\mathrm{m02}$ D & 39.36 & 38.60 & 0.76 & 39.00 & 0.26 & 39.88 & 38.87 & 1.01 & 39.56 & 0.30 \\
\hline $\mathrm{m02} \mathrm{E}$ & 53.93 & 52.17 & 1.76 & 52.92 & 0.52 & 53.48 & 52.30 & 1.18 & 52.99 & 0.35 \\
\hline $\mathrm{m03} \mathrm{A}$ & 37.48 & 36.55 & 0.93 & 36.87 & 0.31 & 36.51 & 35.99 & 0.52 & 36.26 & 0.18 \\
\hline $\mathrm{m03} B$ & 45.96 & 45.17 & 0.79 & 45.62 & 0.28 & 45.64 & 44.78 & 0.86 & 45.00 & 0.25 \\
\hline $\mathrm{m03C}$ & 35.14 & 33.08 & 2.06 & 33.85 & 0.63 & 34.97 & 33.24 & 1.73 & 34.33 & 0.46 \\
\hline m03 D & 37.59 & 36.18 & 1.41 & 37.19 & 0.45 & 38.02 & 37.30 & 0.72 & 37.60 & 0.22 \\
\hline m03 E & 48.34 & 47.35 & 0.99 & 47.78 & 0.28 & 48.43 & 47.45 & 0.98 & 47.90 & 0.31 \\
\hline $\mathrm{m04} \mathrm{A}$ & - & - & - & - & - & - & - & - & - & - \\
\hline m04 B & 47.26 & 46.64 & 0.62 & 46.90 & 0.20 & 46.88 & 46.17 & 0.71 & 46.48 & 0.21 \\
\hline m04 C & 35.52 & 33.34 & 2.18 & 34.07 & 0.62 & 35.41 & 34.38 & 1.03 & 34.81 & 0.32 \\
\hline m04 D & 36.68 & 35.88 & 0.80 & 36.36 & 0.28 & 37.36 & 37.05 & 0.31 & 37.18 & 0.12 \\
\hline m05 A & 31.70 & 29.43 & 2.27 & 30.92 & 0.60 & 30.74 & 29.67 & 1.07 & 30.25 & 0.31 \\
\hline m05 B & 42.88 & 42.23 & 0.65 & 42.53 & 0.22 & 43.35 & 42.34 & 1.01 & 42.71 & 0.32 \\
\hline m05 C & 32.89 & 31.19 & 1.70 & 32.41 & 0.46 & 34.26 & 33.84 & 0.42 & 34.09 & 0.14 \\
\hline m05 D & 34.00 & 32.09 & 1.91 & 33.45 & 0.57 & 34.58 & 33.73 & 0.85 & 34.19 & 0.25 \\
\hline m05 E & 43.69 & 42.77 & 0.92 & 43.15 & 0.29 & 44.08 & 43.43 & 0.65 & 43.79 & 0.21 \\
\hline $\mathrm{m06} \mathrm{A}$ & 37.70 & 36.31 & 1.39 & 36.86 & 0.49 & 36.08 & 35.33 & 0.75 & 35.80 & 0.22 \\
\hline m06 B & 52.00 & 51.40 & 0.60 & 51.61 & 0.17 & 52.83 & 51.92 & 0.91 & 52.33 & 0.30 \\
\hline $\mathrm{m06C}$ & 38.71 & 38.26 & 0.45 & 38.43 & 0.55 & 39.55 & 38.31 & 1.24 & 38.78 & 0.37 \\
\hline m06 D & 40.09 & 39.20 & 0.89 & 39.64 & 0.28 & 40.64 & 39.70 & 0.94 & 40.24 & 0.29 \\
\hline $\mathrm{m} 06 \mathrm{E}$ & 53.89 & 53.51 & 0.38 & 53.69 & 0.12 & 54.95 & 53.87 & 1.08 & 54.26 & 0.32 \\
\hline $\mathrm{m} 07 \mathrm{~A}$ & 30.60 & 30.30 & 0.30 & 30.44 & 0.09 & 30.97 & 30.50 & 0.47 & 30.75 & 0.16 \\
\hline m07 B & 40.17 & 39.02 & 1.15 & 39.69 & 0.34 & 40.01 & 39.36 & 0.65 & 39.71 & 0.24 \\
\hline $\mathrm{m07C}$ & 30.17 & 29.84 & 0.33 & 30.06 & 0.10 & 31.45 & 30.95 & 0.50 & 31.20 & 0.15 \\
\hline m07 D & 36.42 & 34.80 & 1.62 & 35.73 & 0.54 & 36.52 & 35.72 & 0.80 & 36.06 & 0.31 \\
\hline $\mathrm{m} 07 \mathrm{E}$ & 49.45 & 48.45 & 1.00 & 49.10 & 0.29 & 49.83 & 48.38 & 1.45 & 48.96 & 0.41 \\
\hline $\mathrm{m08A}$ & 35.06 & 34.64 & 0.42 & 34.86 & 0.14 & 34.54 & 33.81 & 0.73 & 34.23 & 0.21 \\
\hline m08 B & 45.54 & 44.12 & 1.42 & 44.75 & 0.42 & 44.63 & 43.72 & 0.91 & 44.22 & 0.25 \\
\hline $\mathrm{m08C}$ & 35.44 & 34.91 & 0.53 & 35.10 & 0.19 & 37.81 & 37.34 & 0.47 & 37.52 & 0.16 \\
\hline m08 D & 34.07 & 33.23 & 0.84 & 33.65 & 0.29 & 34.37 & 33.49 & 0.88 & 33.99 & 0.27 \\
\hline $\mathrm{m} 08 \mathrm{E}$ & 47.38 & 46.10 & 1.28 & 46.84 & 0.43 & 48.19 & 37.81 & 10.38 & 46.65 & 3.12 \\
\hline $\mathrm{m09} \mathrm{A}$ & 38.37 & 37.67 & 0.70 & 38.02 & 0.28 & 37.45 & 36.31 & 1.14 & 37.05 & 0.31 \\
\hline m09 B & 50.48 & 49.75 & 0.73 & 50.24 & 0.23 & 50.93 & 49.89 & 1.04 & 50.42 & 0.38 \\
\hline
\end{tabular}


Table 3. Measurements performed by observer \#2 - cont.

\begin{tabular}{|c|c|c|c|c|c|c|c|c|c|c|}
\hline \multirow{3}{*}{$\begin{array}{l}\text { Measurement } \\
\text { name }\end{array}$} & \multicolumn{10}{|c|}{ Observer \#2 } \\
\hline & \multicolumn{5}{|c|}{$\begin{array}{c}\text { caliper measurement } \\
\text { plaster cast }\end{array}$} & \multicolumn{5}{|c|}{$\begin{array}{l}\text { 3D virtual model measurement } \\
\text { laser scanner }\end{array}$} \\
\hline & $\begin{array}{c}\text { maximal } \\
\text { value (max) } \\
{[\mathrm{mm}]}\end{array}$ & $\begin{array}{c}\text { minimal } \\
\text { value (min) } \\
{[\mathrm{mm}]}\end{array}$ & $\begin{array}{l}\text { difference } \\
\quad(\max - \\
\min )[\mathrm{mm}]\end{array}$ & $\begin{array}{l}\text { mean } \\
\text { value } \\
{[\mathrm{mm}]}\end{array}$ & $\begin{array}{l}\text { standard } \\
\text { deviation } \\
{[\mathrm{mm}]}\end{array}$ & $\begin{array}{c}\text { maximal } \\
\text { value (max) } \\
{[\mathrm{mm}]}\end{array}$ & $\begin{array}{c}\text { minimal } \\
\text { value } \\
\text { (min) }[\mathrm{mm}]\end{array}$ & $\begin{array}{c}\text { difference } \\
{[\mathrm{max}-} \\
\mathrm{min}][\mathrm{mm}]\end{array}$ & $\begin{array}{l}\text { mean } \\
\text { value } \\
{[\mathrm{mm}]}\end{array}$ & $\begin{array}{c}\text { standard } \\
\text { deviation } \\
{[\mathrm{mm}]}\end{array}$ \\
\hline m09 E & 44.21 & 43.38 & 0.83 & 43.93 & 0.27 & 44.38 & 43.89 & 0.49 & 44.12 & 0.15 \\
\hline $\mathrm{m} 10 \mathrm{~A}$ & 40.67 & 39.95 & 0.72 & 40.35 & 0.24 & 41.22 & 40.82 & 0.40 & 41.01 & 0.13 \\
\hline m10 B & 50.38 & 49.68 & 0.70 & 50.06 & 0.21 & 50.95 & 50.31 & 0.64 & 50.60 & 0.22 \\
\hline $\mathrm{m} 10 \mathrm{C}$ & 38.83 & 37.91 & 0.92 & 38.35 & 0.34 & 39.85 & 39.48 & 0.37 & 39.67 & 0.14 \\
\hline m10 D & 38.50 & 38.00 & 0.50 & 38.30 & 0.16 & 37.93 & 37.65 & 0.28 & 37.77 & 0.08 \\
\hline $\mathrm{m} 10 \mathrm{E}$ & 48.41 & 47.78 & 0.63 & 48.17 & 0.17 & 48.23 & 47.26 & 0.97 & 47.58 & 0.29 \\
\hline m11 A & 36.04 & 35.00 & 1.04 & 35.28 & 0.31 & 35.54 & 34.90 & 0.64 & 35.22 & 0.24 \\
\hline m11 B & 46.00 & 45.41 & 0.59 & 45.79 & 0.18 & 46.07 & 45.51 & 0.56 & 45.78 & 0.15 \\
\hline $\mathrm{m} 11 \mathrm{C}$ & 32.00 & 31.52 & 0.48 & 31.78 & 0.17 & 34.10 & 33.58 & 0.52 & 33.87 & 0.16 \\
\hline m11 D & 36.26 & 34.07 & 2.19 & 34.46 & 0.66 & 34.52 & 33.36 & 1.16 & 33.98 & 0.45 \\
\hline $\mathrm{m} 11 \mathrm{E}$ & 46.32 & 45.68 & 0.64 & 45.99 & 0.18 & 46.53 & 45.89 & 0.64 & 46.18 & 0.23 \\
\hline $\mathrm{m} 12 \mathrm{~A}$ & 47.62 & 46.23 & 1.39 & 46.79 & 0.43 & 46.33 & 45.70 & 0.63 & 45.92 & 0.20 \\
\hline $\mathrm{m} 12 \mathrm{~B}$ & 56.67 & 55.87 & 0.80 & 56.40 & 0.25 & 56.81 & 56.00 & 0.81 & 56.50 & 0.26 \\
\hline $\mathrm{m} 12 \mathrm{C}$ & 43.61 & 42.56 & 1.05 & 43.00 & 0.31 & 44.89 & 43.35 & 1.54 & 44.18 & 0.42 \\
\hline m12 D & 45.77 & 44.85 & 0.92 & 45.27 & 0.39 & 45.21 & 44.81 & 0.40 & 45.07 & 0.12 \\
\hline $\mathrm{m} 12 \mathrm{E}$ & 59.52 & 58.96 & 0.56 & 59.24 & 0.19 & 59.77 & 59.35 & 0.42 & 59.57 & 0.14 \\
\hline m13 A & 33.36 & 31.41 & 1.95 & 32.76 & 0.55 & 32.94 & 32.34 & 0.60 & 32.60 & 0.17 \\
\hline m13 B & 46.41 & 45.58 & 0.83 & 46.01 & 0.27 & 46.56 & 45.50 & 1.06 & 45.99 & 0.31 \\
\hline m13 C & 32.37 & 31.90 & 0.47 & 32.14 & 0.14 & 34.42 & 33.78 & 0.64 & 34.17 & 0.20 \\
\hline m13 D & 38.25 & 36.93 & 1.32 & 37.52 & 0.37 & 38.46 & 37.92 & 0.54 & 38.11 & 0.18 \\
\hline m13 E & 56.02 & 55.34 & 0.68 & 55.61 & 0.22 & 56.39 & 55.44 & 0.95 & 55.80 & 0.27 \\
\hline m14 A & 34.60 & 34.11 & 0.49 & 34.34 & 0.15 & 34.62 & 33.99 & 0.63 & 34.29 & 0.21 \\
\hline m14 B & 45.93 & 45.53 & 0.40 & 45.73 & 0.13 & 45.75 & 45.21 & 0.54 & 45.42 & 0.16 \\
\hline $\mathrm{m} 14 \mathrm{C}$ & 32.35 & 31.63 & 0.72 & 32.11 & 0.25 & 34.36 & 33.90 & 0.46 & 34.13 & 0.13 \\
\hline m14 D & 38.43 & 37.45 & 0.98 & 37.93 & 0.32 & 38.46 & 37.61 & 0.85 & 37.98 & 0.25 \\
\hline $\mathrm{m} 14 \mathrm{E}$ & 51.52 & 50.98 & 0.54 & 51.28 & 0.17 & 51.60 & 51.02 & 0.58 & 51.24 & 0.20 \\
\hline
\end{tabular}

Our results were difficult to compare with reports in the literature because studies comparing plaster and digital dental models used considerably different methodologies, with variable numbers of observers, observations and repetitions of measurements, as well as using different types of digital calipers, laser scans, file formats, and software for reconstruction and analysis. ${ }^{10}$ Better standardization is required in order to compare studies and to find stronger evidence for the accuracy of digital models. Moreover, even though measurement with caliper on a plaster cast is recognized as the gold standard, we also found errors in the measurements using this method, which follows the same pattern as those performed using digital models. A methodological alternative may be a comparison of measurements of digital models using a validated industrial laser scanner (gold standard) and a low-cost laser scanner using the same software for measurements.
In conclusion, the null hypothesis was partially accepted. Three-dimensional virtual models from the low-cost DAVID laser scanner can be used clinically, but only for certain types of measurements (types A, B and C). The lowcost DAVID laser scanner cannot be used clinically for measurements related to interproximal contact points. Therefore, the DAVID laser scanner is not suitable for analyses of teeth width, such as Bolton analysis. ${ }^{11}$

\section{ORCID iDs}

Raphael Olszewski (1) https://orcid.org/0000-0002-2211-7731 Joanna Szyper-Szczurowska (1) https://orcid.org/0000-0003-3354-1287 Maciej Opach (1) https://orcid.org/0000-0003-0728-5945 Piotr Bednarczyk (1) https://orcid.org/0000-0003-3725-5894 Jan Zapala (1) https://orcid.org/0000-0002-5439-9614 Stefan Szczepanik (1) https://orcid.org/0000-0001-8680-7750 
Table 4. Comparison of the measurements between observers \#1 and \#2 and the confidence interval with $a=0.05$

\begin{tabular}{|c|c|c|c|c|c|c|}
\hline \multirow{3}{*}{$\begin{array}{l}\text { Measurement } \\
\text { name }\end{array}$} & \multirow{2}{*}{\multicolumn{2}{|c|}{$\begin{array}{l}\text { Difference of mean values } \\
\text { observer \#1 - observer \#2 } \\
\text { [mm] }\end{array}$}} & \multicolumn{4}{|c|}{ Mean value [mm] } \\
\hline & & & \multicolumn{2}{|c|}{ caliper measurement } & \multicolumn{2}{|c|}{ 3D virtual model measurement } \\
\hline & $\begin{array}{l}\text { caliper } \\
\text { measurement }\end{array}$ & $\begin{array}{l}\text { 3D virtual model } \\
\text { measurement }\end{array}$ & observer \#1 & observer \#2 & observer \#1 & observer \#2 \\
\hline $\mathrm{m} 01 \mathrm{~A}$ & 0.58 & -0.08 & $32.81 \pm 0.054$ & $33.39 \pm 0.06$ & $32.92 \pm 0.21$ & $32.85 \pm 0.11$ \\
\hline $\mathrm{m} 01 \mathrm{~B}$ & 0.23 & 0.37 & $46.98 \pm 0.07$ & $47.22 \pm 0.06$ & $47.09 \pm 0.20$ & $47.46 \pm 0.12$ \\
\hline $\mathrm{m} 01 \mathrm{C}$ & -0.40 & 0.18 & $34.43 \pm 0.11$ & $34.03 \pm 0.09$ & $35.27 \pm 0.16$ & $35.45 \pm 0.15$ \\
\hline m01 D & 1.49 & 1.62 & $33.29 \pm 0.05$ & $34.78 \pm 0.12$ & $33.49 \pm 0.14$ & $35.12 \pm 0.12$ \\
\hline $\mathrm{m} 01 \mathrm{E}$ & 2.73 & 2.64 & $42.93 \pm 0.11$ & $45.66 \pm 0.06$ & $43.20 \pm 0.18$ & $45.84 \pm 0.14$ \\
\hline $\mathrm{m02} \mathrm{A}$ & 1.54 & 0.17 & $38.51 \pm 0.06$ & $40.04 \pm 0.14$ & $38.70 \pm 0.19$ & $38.86 \pm 0.20$ \\
\hline $\mathrm{m} 02 \mathrm{~B}$ & 0.33 & -0.19 & $51.78 \pm 0.06$ & $52.11 \pm 0.03$ & $52.46 \pm 0.20$ & $52.27 \pm 0.17$ \\
\hline $\mathrm{m} 02 \mathrm{C}$ & 0.15 & 0.41 & $40.02 \pm 0.15$ & $40.17 \pm 0.05$ & $40.92 \pm 0.32$ & $41.33 \pm 0.17$ \\
\hline $\mathrm{m02} \mathrm{E}$ & 2.81 & 3.09 & $50.11 \pm 0.10$ & $52.92 \pm 0.16$ & $49.90 \pm 0.18$ & $52.99 \pm 0.25$ \\
\hline $\mathrm{m03} \mathrm{A}$ & 0.82 & -0.27 & $36.05 \pm 0.10$ & $36.87 \pm 0.09$ & $36.52 \pm 0.25$ & $36.26 \pm 0.12$ \\
\hline m03 B & 1.12 & 0.12 & $44.50 \pm 0.07$ & $45.62 \pm 0.08$ & $44.89 \pm 0.18$ & $45.00 \pm 0.17$ \\
\hline $\mathrm{m03C}$ & 1.76 & 0.83 & $32.09 \pm 0.30$ & $33.85 \pm 0.19$ & $33.50 \pm 0.17$ & $34.33 \pm 0.32$ \\
\hline m03 D & 2.41 & 1.67 & $34.77 \pm 0.28$ & $37.19 \pm 0.14$ & $35.94 \pm 0.42$ & $37.60 \pm 0.15$ \\
\hline m03 E & 2.43 & 2.36 & $45.36 \pm 0.22$ & $47.78 \pm 0.08$ & $45.53 \pm 0.30$ & $47.90 \pm 0.22$ \\
\hline $\mathrm{m04} \mathrm{A}$ & - & - & - & - & - & - \\
\hline m04 B & 0.64 & 0.05 & $46.26 \pm 0.28$ & $46.90 \pm 0.06$ & $46.43 \pm 0.20$ & $46.48 \pm 0.15$ \\
\hline m04C & 0.53 & 0.36 & $33.54 \pm 0.40$ & $34.07 \pm 0.19$ & $34.44 \pm 0.25$ & $34.81 \pm 0.22$ \\
\hline m04 D & 1.48 & 0.77 & $34.88 \pm 0.31$ & $36.36 \pm 0.08$ & $36.41 \pm 0.13$ & $37.18 \pm 0.08$ \\
\hline $\mathrm{m04E}$ & 3.54 & 4.34 & $48.84 \pm 0.21$ & $52.37 \pm 0.08$ & $48.94 \pm 0.21$ & $53.28 \pm 0.15$ \\
\hline m05 B & 0.26 & -0.06 & $42.27 \pm 0.27$ & $42.53 \pm 0.01$ & $42.76 \pm 0.24$ & $42.71 \pm 0.22$ \\
\hline m05 C & 0.12 & 0.73 & $32.29 \pm 0.25$ & $32.41 \pm 0.14$ & $33.36 \pm 0.22$ & $34.09 \pm 0.10$ \\
\hline m05 D & 2.87 & 3.06 & $30.58 \pm 0.26$ & $33.45 \pm 0.18$ & $31.14 \pm 0.32$ & $34.19 \pm 0.17$ \\
\hline m05 E & 1.91 & 2.10 & $41.24 \pm 0.29$ & $43.15 \pm 0.09$ & $41.70 \pm 0.22$ & $43.79 \pm 0.15$ \\
\hline $\mathrm{m06} \mathrm{A}$ & 1.60 & 0.35 & $35.26 \pm 0.21$ & $36.86 \pm 0.15$ & $35.45 \pm 0.21$ & $35.80 \pm 0.15$ \\
\hline m06 B & 0.20 & 0.65 & $51.41 \pm 0.14$ & $51.61 \pm 0.05$ & $51.68 \pm 0.19$ & $52.33 \pm 0.21$ \\
\hline $\mathrm{m06C}$ & -0.15 & -0.64 & $38.58 \pm 0.20$ & $35.43 \pm 0.00$ & $39.43 \pm 0.24$ & $38.78 \pm 0.26$ \\
\hline m06 D & 2.57 & 2.02 & $37.07 \pm 0.23$ & $39.64 \pm 0.08$ & $38.22 \pm 0.28$ & $40.24 \pm 0.20$ \\
\hline $\mathrm{m06} \mathrm{E}$ & 2.54 & 2.59 & $51.15 \pm 0.20$ & $53.69 \pm 0.03$ & $51.67 \pm 0.22$ & $54.26 \pm 0.22$ \\
\hline $\mathrm{m} 07 \mathrm{~A}$ & 0.51 & 0.18 & $29.93 \pm 0.07$ & $30.44 \pm 0.02$ & $30.56 \pm 0.32$ & $30.75 \pm 0.11$ \\
\hline m07 B & -0.01 & -0.03 & $39.69 \pm 0.14$ & $39.69 \pm 0.10$ & $39.74 \pm 0.28$ & $39.71 \pm 0.17$ \\
\hline $\mathrm{m07C}$ & 0.57 & 0.14 & $29.49 \pm 0.31$ & $30.06 \pm 0.03$ & $31.06 \pm 0.23$ & $31.20 \pm 0.10$ \\
\hline m07 D & 1.17 & 1.06 & $34.56 \pm 0.23$ & $35.73 \pm 0.17$ & $35.00 \pm 0.32$ & $36.06 \pm 0.22$ \\
\hline $\mathrm{m} 07 \mathrm{E}$ & 2.77 & 1.39 & $46.33 \pm 0.18$ & $49.10 \pm 0.09$ & $47.58 \pm 0.30$ & $48.96 \pm 0.29$ \\
\hline $\mathrm{m08A}$ & 0.63 & -0.32 & $34.23 \pm 0.27$ & $34.86 \pm 0.04$ & $34.55 \pm 0.16$ & $34.23 \pm 0.15$ \\
\hline $\mathrm{m08B}$ & 0.37 & -0.30 & $44.39 \pm 0.20$ & $44.75 \pm 0.13$ & $44.52 \pm 0.15$ & $44.22 \pm 0.17$ \\
\hline $\mathrm{m08C}$ & 0.89 & 1.21 & $34.20 \pm 0.44$ & $35.10 \pm 0.06$ & $36.30 \pm 0.20$ & $37.52 \pm 0.11$ \\
\hline m08 D & 1.52 & 1.15 & $32.13 \pm 0.22$ & $33.65 \pm 0.09$ & $32.84 \pm 0.39$ & $33.99 \pm 0.19$ \\
\hline $\mathrm{m08E}$ & 2.42 & 1.55 & $44.41 \pm 0.32$ & $46.84 \pm 0.13$ & $45.10 \pm 0.18$ & $46.65 \pm 0.23$ \\
\hline m09 A & 1.61 & 0.59 & $36.42 \pm 0.18$ & $38.02 \pm 0.08$ & $36.47 \pm 0.27$ & $37.05 \pm 0.22$ \\
\hline m09 B & 0.01 & 0.49 & $50.23 \pm 0.22$ & $50.24 \pm 0.07$ & $49.93 \pm 0.25$ & $50.42 \pm 0.27$ \\
\hline m09C & 0.35 & 0.54 & $37.87 \pm 0.18$ & $38.22 \pm 0.11$ & $39.11 \pm 0.22$ & $39.65 \pm 0.05$ \\
\hline
\end{tabular}


Table 4. Comparison of the measurements between observers \#1 and \#2 and the confidence interval with $a=0.05-$ cont.

\begin{tabular}{|c|c|c|c|c|c|c|}
\hline \multirow{3}{*}{$\begin{array}{l}\text { Measurement } \\
\text { name }\end{array}$} & \multirow{2}{*}{\multicolumn{2}{|c|}{$\begin{array}{l}\text { Difference of mean values } \\
\text { observer \#1 - observer \#2 } \\
\text { [mm] }\end{array}$}} & \multicolumn{4}{|c|}{ Mean value $[\mathrm{mm}]$} \\
\hline & & & \multicolumn{2}{|c|}{ caliper measurement } & \multicolumn{2}{|c|}{ 3D virtual model measurement } \\
\hline & $\begin{array}{c}\text { caliper } \\
\text { measurement }\end{array}$ & $\begin{array}{l}\text { 3D virtual model } \\
\text { measurement }\end{array}$ & observer \#1 & observer \#2 & observer \#1 & observer \#2 \\
\hline m09E & 2.55 & -0.40 & $41.38 \pm 0.29$ & $43.93 \pm 0.08$ & $44.51 \pm 0.18$ & $44.12 \pm 0.10$ \\
\hline $\mathrm{m} 10 \mathrm{~A}$ & 0.76 & 0.19 & $39.59 \pm 0.12$ & $40.35 \pm 0.07$ & $40.82 \pm 0.17$ & $41.01 \pm 0.09$ \\
\hline $\mathrm{m} 10 \mathrm{~B}$ & 0.63 & 0.22 & $49.44 \pm 0.14$ & $50.06 \pm 0.06$ & $50.38 \pm 0.22$ & $50.60 \pm 0.15$ \\
\hline $\mathrm{m} 10 \mathrm{C}$ & 0.51 & 1.35 & $37.84 \pm 0.33$ & $38.35 \pm 0.06$ & $38.32 \pm 0.20$ & $39.67 \pm 0.10$ \\
\hline m10 D & 4.19 & 2.34 & $34.11 \pm 0.44$ & $38.30 \pm 0.05$ & $35.43 \pm 0.31$ & $37.77 \pm 0.05$ \\
\hline $\mathrm{m} 10 \mathrm{E}$ & 2.62 & 1.76 & $45.55 \pm 0.15$ & $48.17 \pm 0.05$ & $45.82 \pm 0.23$ & $47.58 \pm 0.26$ \\
\hline $\mathrm{m} 11 \mathrm{~A}$ & 1.33 & 0.82 & $33.95 \pm 0.28$ & $35.28 \pm 0.09$ & $34.39 \pm 0.25$ & $35.22 \pm 0.17$ \\
\hline m11 B & 0.70 & 0.07 & $45.08 \pm 0.02$ & $45.79 \pm 0.05$ & $45.71 \pm 0.25$ & $45.78 \pm 0.10$ \\
\hline $\mathrm{m} 11 \mathrm{C}$ & -0.14 & 1.59 & $31.92 \pm 0.20$ & $31.78 \pm 0.05$ & $32.27 \pm 0.33$ & $33.87 \pm 0.11$ \\
\hline m11 D & 3.64 & 1.97 & $30.81 \pm 0.30$ & $34.46 \pm 0.05$ & $32.01 \pm 0.32$ & $33.98 \pm 0.22$ \\
\hline $\mathrm{m} 11 \mathrm{E}$ & 2.20 & 2.63 & $43.78 \pm 0.32$ & $45.99 \pm 0.20$ & $43.56 \pm 0.26$ & $46.18 \pm 0.16$ \\
\hline $\mathrm{m} 12 \mathrm{~A}$ & 1.33 & -0.13 & $45.47 \pm 0.10$ & $46.79 \pm 0.13$ & $46.06 \pm 0.16$ & $45.92 \pm 0.14$ \\
\hline $\mathrm{m} 12 \mathrm{~B}$ & 1.11 & 0.66 & $55.29 \pm 0.17$ & $56.40 \pm 0.07$ & $55.84 \pm 0.18$ & $56.50 \pm 0.18$ \\
\hline $\mathrm{m} 12 \mathrm{C}$ & 0.35 & 0.76 & $42.65 \pm 0.35$ & $43.00 \pm 0.09$ & $43.42 \pm 0.30$ & $44.18 \pm 0.30$ \\
\hline m12 D & 3.11 & 2.49 & $42.16 \pm 0.30$ & $45.27 \pm 0.12$ & $42.58 \pm 0.22$ & $45.07 \pm 0.08$ \\
\hline $\mathrm{m} 12 \mathrm{E}$ & 2.55 & 2.79 & $56.69 \pm 0.22$ & $59.24 \pm 0.06$ & $56.78 \pm 0.28$ & $59.57 \pm 0.10$ \\
\hline m13 A & 1.03 & 0.81 & $31.73 \pm 0.19$ & $32.76 \pm 0.17$ & $31.78 \pm 0.35$ & $32.60 \pm 0.12$ \\
\hline m13 B & 0.93 & 1.18 & $45.08 \pm 0.20$ & $46.01 \pm 0.08$ & $44.81 \pm 0.17$ & $45.99 \pm 0.22$ \\
\hline $\mathrm{m} 13 \mathrm{C}$ & -0.15 & 0.44 & $32.29 \pm 0.22$ & $32.14 \pm 0.04$ & $33.73 \pm 0.24$ & $34.17 \pm 0.14$ \\
\hline m13 D & 1.76 & 1.48 & $35.77 \pm 0.23$ & $37.52 \pm 0.11$ & $36.63 \pm 0.31$ & $38.11 \pm 0.12$ \\
\hline m13 E & 3.14 & 2.88 & $52.48 \pm 0.23$ & $55.61 \pm 0.06$ & $52.92 \pm 0.37$ & $55.80 \pm 0.19$ \\
\hline m14 A & 1.35 & 0.21 & $32.99 \pm 0.21$ & $34.34 \pm 0.04$ & $34.08 \pm 0.17$ & $34.29 \pm 0.15$ \\
\hline m14 B & 0.92 & 0.27 & $44.81 \pm 0.22$ & $45.73 \pm 0.04$ & $45.15 \pm 0.17$ & $45.42 \pm 0.11$ \\
\hline $\mathrm{m} 14 \mathrm{C}$ & 0.28 & 0.85 & $31.83 \pm 0.37$ & $32.11 \pm 0.07$ & $33.29 \pm 0.33$ & $34.13 \pm 0.09$ \\
\hline m14 D & 2.52 & 1.31 & $35.41 \pm 0.25$ & $37.93 \pm 0.10$ & $36.67 \pm 0.30$ & $37.98 \pm 0.17$ \\
\hline $\mathrm{m} 14 \mathrm{E}$ & 3.24 & 4.09 & $48.05 \pm 0.37$ & $51.28 \pm 0.05$ & $47.16 \pm 0.62$ & $51.24 \pm 0.14$ \\
\hline
\end{tabular}

Table 5. Threshold differences of the measurements between the 2 observers and the 2 methods

\begin{tabular}{|c|c|c|c|c|c|c|}
\hline Threshold [mm] & Method of measurement & Measurement A & Measurement B & Measurement C & Measurement D & Measurement E \\
\hline \multirow{2}{*}{$<0.5$} & plaster cast & 0 & 7 & 9 & 0 & 0 \\
\hline & laser scan & 10 & 11 & 5 & 0 & 1 \\
\hline \multirow{2}{*}{$0.51-1.00$} & plaster cast & 5 & 5 & 4 & 0 & 0 \\
\hline & laser scan & 4 & 2 & 6 & 1 & 0 \\
\hline \multirow{2}{*}{$1.01-1.50$} & plaster cast & 6 & 2 & 0 & 3 & 0 \\
\hline & laser scan & 0 & 1 & 2 & 5 & 1 \\
\hline \multirow{2}{*}{$1.51-2.00$} & plaster cast & 3 & 0 & 1 & 3 & 1 \\
\hline & laser scan & 0 & 0 & 1 & 3 & 2 \\
\hline \multirow{2}{*}{$>2$} & plaster cast & 0 & 0 & 0 & 8 & 13 \\
\hline & laser scan & 0 & 0 & 0 & 5 & 10 \\
\hline
\end{tabular}




\section{References}

1. Jiménez-Gayosso SI, Lara-Carrillo E, López-González S, et al. Difference between manual and digital measurements of dental arches of orthodontic patients. Medicine (Baltimore). 2018;97(22):e10887.

2. Reuschl RP, Heuer W, Stiesch M, Wenzel D, Dittmer MP. Reliability and validity of measurements on digital study models and plaster models. Eur J Orthod. 2016;38(1):22-26.

3. Peluso MJ, Josell SD, Levine SW, Lorei BJ. Digital models: An introduction. Semin Orthod. 2004;10(3):226-238.

4. Nowak R, Wesemann C, Robben J, Muallah J, Bumann A. An in-vitro study comparing the accuracy of full-arch casts digitized with desktop scanners. Quintessence Int. 2017;20:667-676.

5. Robben J, Muallah J, Wesemann C, et al. Suitability and accuracy of CBCT model scan: An in vitro study. Int J Comput Dent. 2017;20(4): 363-375.

6. Goracci C, Franchi L, Vichi A, Ferrari M. Accuracy, reliability, and efficiency of intraoral scanners for full-arch impressions: A systematic review of the clinical evidence. Eur J Orthod. 2016;38(4):422-428.
7. Howe RP, McNamara JA, Jr, O'Connor KA. An examination of dental crowding and its relationship to tooth size and arch dimension. Am J Orthod. 1983;83(5):363-373.

8. Horton HM, Miller JR, Gaillard PR, Larson BE. Technique comparison for efficient orthodontic tooth measurements using digital models. Angle Orthod. 2010;80(2):254-261.

9. Kim J, Lagravére MO. Accuracy of Bolton analysis measured in laser scanned digital models compared with plaster models (gold standard) and cone-beam computer tomography images. Korean JOrthod. 2016;46(1):13-19.

10. De Luca Canto G, Pachêco-Pereira C, Lagravere MO, Flores-Mir C, Major PW. Intra-arch dimensional measurement validity of laserscanned digital dental models compared with the original plaster models: A systematic review. Orthod Craniofac Res. 2015;18(2):65-76.

11. Bailey E, Nelson G, Miller AJ, Andrews L, Johnson E. Predicting toothsize discrepancy: A new formula utilizing revised landmarks and 3-dimensional laser scanning technology. Am J Orthod Dentofacial Orthop. 2013;143(4):574-585. 